\title{
Editorial to Special Issue on Deep Learning for Intelligent Multimedia Analytics
}

This special issue is highly motivated by the convergence of deep learning and multi-modal contents, since digital contents nowadays are inherently multimedia and the advancement of deep learning has greatly changed the landscape of multimedia content analysis. Multimedia data has been generated, published, and spread explosively, becoming an indispensable part of today's big data. Such large-scale multimedia data has created challenges and opportunities for intelligent multimedia analysis. Meanwhile, with the recent advances in deep-learning techniques, we are now able to boost the intelligent multimedia analysis significantly. As a result, deeplearning-based intelligent multimedia analysis is becoming an emerging research area in the field of multimedia.

The aim of this special issue is to call for a coordinated effort to understand the scenarios and challenges emerging in multimedia analysis with deep-learning techniques. The multimedia data of interest cover a wide spectrum, ranging from text, audio, image, and web videos to surveillance videos. Particularly, this special issue focuses on the visual domain, e.g., image and video analysis. Articles included cover a variety of topics, including representation learning, recognition, as well as real applications driven by deep-based techniques.

The first article of this special issue, authored by the guest editors, presents a survey on deeplearning-based multimedia analytics, which starts by summarizing the fundamental deep-learning techniques and then reviews representative high-level tasks such as classification, detection, captioning, and segmentation. Progress on popular benchmarks gives a clear path of development and helps identify the milestone works and future directions.

The following articles in this special issue introduce a variety of deep based multimedia analytics solutions. Most of the articles in this special issue address the problem of recognition and representation learning.

The article titled "Convolutional Attention Networks for Scene Text Recognition," by Shancheng Fang, Hongtao Xie, Yating Yang, Yan Li, and Yongdong Zhang, presents a convolutional attention network (CAN) for unconstrained scene text recognition based on a fully Convolutional Neural Network (CNN) architecture and the attention mechanism.

The article "Structure-Aware Deep Learning for Product Image Classification," by Zhineng Chen, Shanshan Ai, and Caiyan Jia, classifies product images by leveraging the hierarchical structure and attributes existing in online web stores. With these structural-aware clues, an advanced CNN model is developed beyond the one-versus-all classification as in conventional methods.

The article "Deep Patch Representations with Shared Codebook for Scene Classification," by Shuqiang Jiang, Gongwei Chen, Xinhang Song, and Linhu Liu, studies the scene classification by deep patch representations. Compared with general objects (e.g., product), scene images are more abstract. Object and scene images have different characteristics with different scales and composition structures. This article particularly studies how to integrate the local mid-level semantic representation, including both object and scene concepts, with shared codebooks.

The article "Visual Content Recognition by Exploiting Semantic Feature Map with Attention and Multi-task Learning," by Rui-wei Zhao, Zuxuan Wu, Jianguo Li, and Yu-gang Jiang, addresses

(C) 2019 Copyright held by the owner/author(s).

1551-6857/2019/01-ART1

https://doi.org/10.1145/3292059

ACM Trans. Multimedia Comput. Commun. Appl., Vol. 15, No. 1s, Article 1. Publication date: January 2019. 
another recognition problem by exploiting the spatial relationships among objects. By learning a Semantic Feature Map (SFM) with the attention-based network, spatial contexts are explicitly modeled. Extensive evaluations and comparative studies verify the proposed approach and very promising results are reported on Pascal VOC and MS-COCO benchmarks.

The article titled "Cross-Modality Feature Learning via Convolutional AutoEncoder," by Xueliang Liu, Meng Wang, Zheng-jun Zha, and Richang Hong, studies the embedding problem, which is also deeply rooted in a classification problem. The authors propose a MUltimodal Convolutional AutoEncoder (MUCAE) approach to learn representative features from visual and textual modalities. Compared to conventional solutions relying on hand-crafted features, MUCAE encodes features from image pixels and text characters directly and produces more representative and robust features.

The article "Dense 3D-Convolutional Neural Network for Person Re-Identification in Videos," by Jiawei Liu, Zheng-jun Zha, Xuejin Chen, Zilei Wang, and Yongdong Zhang, addresses person re-identification problems in videos. This article is also related to representation learning. The authors propose a Dense 3D-Convolutional Network (D3DNet) to jointly learn spatio-temporal and appearance features. The 3D dense blocks enlarge the receptive fields of visual neurons in spatial and temporal dimensions, leading to discriminative appearance representation as well as short-term and long-term motion information of pedestrians.

The article "Deep Semantic Mapping for Heterogeneous Multimedia Transfer Learning Using Co-Occurrence Data," by Liang Zhao, Zhikui Chen, Laurence Wang, Jamal Deen, and Jane Wang, also studies the representation learning problem in a transfer learning setting. The authors propose a deep semantic mapping model for heterogeneous multimedia transfer learning (DHTL) using co-occurrence data, where a multi-layer correlation matching network across domains is constructed. canonical correlation analysis (CCA) is combined to bridge each pair of domainspecific hidden layers. Extensive experiments for three multimedia recognition applications demonstrate the effectiveness of the proposed DHTL.

The article "Applying Deep Learning for Epilepsy Seizure Detection and Brain Mapping Visualization," by Shamim Hossain, Syed Umar Amin, Mansour Alsulaiman, and Ghulam Muhammad, explores the deep based techniques in medical domain. Seizures are hard to detect, as they vary a lot for inter- and intra-patients. This article proposes a deep CNN model for seizure detection on an open-access electroencephalogram (EEG) epilepsy dataset collected at the Boston Children's Hospital. The visualized result can be used as an effective multimedia tool for producing quick and relevant brain-mapping images utilizable by medical experts for further investigation.

\author{
Wei Zhang \\ JD AI Research \\ Ting Yao \\ JD AI Research \\ Shiai Zhu \\ Alibaba Group \\ Abdulmotaleb El Saddik \\ University of Ottawa
}

Guest Editors 